hep-th/0503243

March 2005

\title{
Induced Curvature in Brane Worlds by Surface Terms in String Effective Actions with Higher-Curvature Corrections
}

\author{
Nick E. Mavromatos ${ }^{a, *}$ and Eleftherios Papantonopoulos ${ }^{b, * *}$ \\ ${ }^{a}$ Physics Department, Theoretical Physics, \\ King's College London, \\ London WC2R 2LS, U.K. \\ ${ }^{b}$ National Technical University of Athens, \\ Physics Department, \\ Zografou Campus, GR 157 80, Athens, Greece.
}

\begin{abstract}
In string-inspired effective actions, representing the low-energy bulk dynamics of brane/string theories, the higher-curvature ghost-free Gauss-Bonnet combination is obtained by local field redefinitions which leave the (perturbative) string amplitudes invariant. We show that such redefinitions lead to surface terms which induce curvature on the brane world boundary of the bulk spacetime.
\end{abstract}

* email address: nikolaos.mavromatos@kcl.ac.uk

** email address: lpapa@central.ntua.gr 


\section{Introduction}

In recent years brane world models attracted a lot of attention of the physics community, as a result of their elegant and novel ways of tackling fundamental issues of particle physics, such as new attempts to solve the hierarchy problem [1], and new approaches to cosmology [2]. By representing our observable world as a thin membrane (brane), in a higher-dimensional spacetime called bulk, one obtains an unconventional way of looking at the relation between gravity and matter. The latter is confined on the brane world, not allowed to propagate on the bulk space, while gravity is free to propagate on the bulk, although in warped geometries [1], localization of gravity on the brane world does occur, in the sense of a peak of the corresponding wavefunction for the graviton mode [3].

Such brane world scenaria appear attractive from various view points. One is the novel mechanisms these models provide in connection with a possible geometric resolution of the hierarchy problem [4], which is represented as a consequence of a certain distance scale of two brane worlds. The other point of view is that of cosmology, since the brane world scenaria provide a variety of new ways of looking at the Physics of the Early Universe, including inflation, and in general cosmological evolution [5].

Other important features of these scenaria include the so-called AdS/CFT correspondence. Indeed, in most of the scenaria discussed in the literature so far, the bulk space appears to be anti-de-Sitter (AdS), with negative bulk cosmological constant. In such models the brane worlds constitute, then, the (single) boundary of the AdS space, and the holographic Maldacena conjecture [6] is in operation: all the information included in the correlation functions of the matter quantum field theory on the boundary of such a space can be obtained by means of a classical (super)gravity theory in the bulk space, which may be the limit of some underlying string/brane/M-theory. In this way, one obtains a holographic image of the bulk space on the boundary, and no information loss could occur, even if the bulk geometry contains singularities, such as black holes etc. The AdS/CFT correspondence certainly provides a completely novel way of dealing with matter quantum field theories on brane worlds. A manifestation of this approach concerns the induced gravity on the brane $[7,8]$ boundary, which results in a curved spacetime boundary quantum field theory. The AdS/CFT correspondence on the other hand, on more phenomenological grounds, may help us, among other things, to understand old unresolved problems of cosmology like the exit from inflation and reheating [9].

A very interesting feature of such brane world models is the fact that the gravitational scale on the brane world is not a fundamental scale of the theory, but it is induced in a variety of ways, and is model dependent. The higher-dimensional bulk gravitational scale is viewed as the fundamental scale, while the four-dimensional Planck scale is defined as the coefficient of the four-dimensional Einstein (scalar curvature) term in the low-energy effective action, describing the induced gravity on the brane world $[7,8]$, even if there are curvature correction terms in the bulk, like Gauss-Bonnet terms [10]. It is important to notice that the precise relation of the four-dimensional Planck scale and the bulk gravitational scale is model dependent, in particular it depends crucially on the form of the higher-dimensional metric which is a solution of the bulk gravitational equations of motion. 
A brane world model that combines both curvature corrections in the five-dimensional action, a Gauss-Bonnet term in the bulk and a scalar curvature term on the brane boundary, was proposed in [11]. An important constraint in such kind of models is the so-called matching conditions of the metric, and other fields propagating in the bulk, at the discontinuous regions ("junctions") of the bulk space defined by the positions of the brane worlds. By requiring smooth functions of these fields at the junctions implies important constraints (Israel boundary conditions), which are quite restrictive for the solutions of the low-energy theory $[10,12,13]$. Recently it has been pointed out [14] that the matching conditions possess ambiguities, in the sense that one may find consistent conditions for arbitrary boundary gravitational actions, i.e. $S_{1}=\int_{\text {brane }} f\left(R^{(b)}\right)$, where $f(\ldots)$ is an arbitrary function of the boundary scalar curvature $R^{(b)}$. Such an arbitrariness persists to all orders in perturbation theory, and includes higher-order curvature corrections in the effective action. This results in the loss of the predictability of the model, as far as the low-energy phenomenology or cosmology are concerned, and thus brings up the necessity of an underlying fundamental theory, which would restrict the form of the boundary and bulk actions.

In this work we will show that strings is a relative simple and natural framework, where such a restriction occurs. Starting with a five-dimensional effective string action including also the Gauss-Bonnet combination of higher-curvature ghost-free terms, and no boundary terms in the first place, we show, that local field redefinitions which leave the (perturbative) string amplitudes invariant, lead to surface terms which induce curvature on the brane world boundary of the bulk spacetime. These terms introduce an energy scale on the brane which depends on the parameters of the bulk. Such terms of course can be generated also by quantum corrections of matter fields on the boundary [7], but we shall not deal with such corrections here

\section{String Amplitudes in the Bulk, Field-Redefinition Ambiguities and Brane Effective Actions}

The string theory requires that the effective low-energy action, in both the bulk and the brane world, admits a systematic derivative expansion in powers of $\alpha^{\prime} p^{2}$, with $p$ a generic momentum scale, in the closed string sector, and a corresponding expansion of $\sqrt{\alpha^{\prime}} p$ in the open string sector on the brane world, where $\alpha^{\prime}=1 / M_{s}^{2}$, with $M_{s}$ the string scale (which is sometimes called bulk gravitational scale). It is important to notice that we use the same string theory, in bulk and brane, in the sense of having a single string coupling and scale. We also use a systematic derivative expansion in both the brane and the bulk. This implies that if we restrict ourselves to quadratic curvature corrections in the low-energy effective action in the bulk, we shall do the same on the brane world as well, since we used momentum scales such that $\sqrt{\alpha^{\prime}} p \ll 1$.

In the bulk this leads to effective actions which contain a derivative expansion up to the desired order. In the gravitational sector, for instance, this leads to several powers 
of curvature tensors. In the present work we shall work up to quadratic power in such curvature tensors, which is equivalent to keeping up to four derivatives in other terms. For our purposes we shall restrict ourselves to the graviton and dilaton fields of the (bosonic part of) the gravitational multiplet of the bulk (super)string. There is an important result in such perturbative calculations, pertaining to ambiguities in several coefficients of such an effective action $[15,16]$, as a result of local field redefinitions (i.e. redefinitions involving positive (or zero) powers of local fields), which leave the scattering amplitudes invariant.

We consider, for definiteness, the case in which the action is in five spacetime dimensions. Some remarks are in order at this point. From a formal point of view, one may think [17] of the (bulk) fifth dimension in the spacetime as a (spacelike) Liouville mode [18]. A more conventional (and probably safer) approach, which we shall adopt here, is to assume initially a ten-dimensional spacetime, in which three branes are embedded. In the bulk one may, then, consider the propagation of closed strings only, but take the case in which all but one of the bulk coordinates are compactified. In that case, the induced string theory amplitudes will formally correspond to those living in an effective five-dimensional spacetime, in the sense that one may consider string backgrounds that depend only on the uncompactified coordinates, and restrict oneself to effective string amplitudes (or, equivalently, $\sigma$-model conformal-invariance conditions) for those degrees of freedom.

The low-energy effective action we consider is of the form

$$
S=S_{5}+S_{4}
$$

where $S_{5}$ denotes bulk contributions, and $S_{4}$ boundary contributions on four-dimensional (three space) boundary domain walls. The four-dimensional part $S_{4}$ of the action (1) is defined as

$$
S_{4}=\sum_{i} \int d^{4} x \sqrt{-g_{(4)}} e^{\omega \Phi} v\left(z_{i}\right)
$$

where $\omega$ is a constant, $\Phi$ is the dilaton field and

$$
g_{(4)}^{\mu \nu}= \begin{cases}g^{\mu \nu} & \mu, \nu<5 \\ 0 & \text { otherwise },\end{cases}
$$

and the sum over $i$ extends over D-brane walls located at $z=z_{i}$ along the fifth dimension. The quantity $v\left(z_{i}\right)$ is the brane tension, which arises also from quantum loop corrections of matter fields localized on the brane.

The five-dimensional $\mathcal{O}\left(\alpha^{\prime}\right)$ ( $\alpha^{\prime}$ the Regge slope) effective action for graviton and dilaton bulk fields reads:

$$
\begin{aligned}
S_{5}= & \int d^{5} x \sqrt{-g}\left[-R-\frac{4}{3}\left(\nabla_{\mu} \Phi\right)^{2}+f(\Phi)\left(\gamma R^{2}+\beta R_{\mu \nu}^{2}+\alpha R_{\mu \nu \rho \sigma}^{2}\right)\right. \\
& \left.+\xi(z) e^{\zeta \Phi}+c_{2} f(\Phi)\left(\nabla_{\mu} \Phi\right)^{4}+\ldots\right]
\end{aligned}
$$

where Greek indices are five-dimensional indices, and the dots denote other types of contraction of the four-derivative dilaton terms; these will not be of interest to us here, for 
reasons that will be explained below. The quantity $\xi(z)$ denotes the bulk cosmological constant, which for simplicity is taken to depend only on the fifth coordinate $z$. We also have

$$
\alpha=+1, \quad f(\Phi)=\lambda e^{\theta \Phi}, \lambda=\alpha^{\prime} / 8 g_{s}^{2}>0
$$

where $g_{s}$ is the string coupling, and $\zeta=-\theta=4 / \sqrt{3(D-2)}(=4 / 3$ in five dimensions of (formal) interest to us here). Moreover, as mentioned above, in (perturbative) string theory one has the freedom [16] to redefine the graviton and dilaton fields so as to ensure that the quadratic-curvature terms in (4) are of the ghost-free GB form [19]

$$
\mathcal{R}_{G B}^{2}=R_{\mu \nu \rho \sigma} R^{\mu \nu \rho \sigma}-4 R_{\mu \nu} R^{\mu \nu}+R^{2}
$$

This field-redefinition ambiguity also allows us to consider the four-derivative dilaton terms in (4) as having the single structure exhibited above. Matching with tree-level string amplitudes to $\mathcal{O}\left(\alpha^{\prime}\right)$ then requires [16]

$$
c_{2}=\frac{16}{9} \frac{D-4}{D-2} .
$$

The pertinent field redefinitions read (for the normalization of the field as they appear in $(4))$

$$
\begin{aligned}
g_{\mu \nu} \rightarrow g_{\mu \nu}^{\prime} & =g_{\mu \nu}+\alpha^{\prime} e^{4 \Phi / \sqrt{3(D-2)}}\left(b_{1} R_{\mu \nu}+b_{2} g_{\mu \nu} R+b_{3} \partial_{\mu} \Phi \partial_{\nu} \Phi+\right. \\
& \left.+b_{4} g_{\mu \nu}(\partial \Phi)^{2}+b_{5} g_{\mu \nu} \square \Phi\right), \\
\Phi \rightarrow \Phi^{\prime} & =\Phi+e^{4 \Phi / \sqrt{3(D-2)}}\left(c_{1}+c_{2}(\partial \Phi)^{2}+c_{3} \square \Phi\right),
\end{aligned}
$$

where $\square$ denotes the covariant D'Alembertian with respect to the old metric $g_{\mu \nu}$, and $b_{i}, c_{i}$ are real constant coefficients. For our purposes, for simplicity and without loss of generality, we shall assume that the initial bulk action has the form (4), that is, not necessarily ghost free form, but with a single structure of dilaton four-derivative terms, parameterized by the coefficient $c_{2}$. This restricts the class of redefinitions we shall discuss in the present article to those involving $b_{1}, b_{2}$ coefficients only.

The corresponding change of the bulk action (4) under such redefinitions may be summarized as follows: the coefficients $\beta$ and $\gamma$ change as

$$
\beta \rightarrow \beta^{\prime}=\beta-b_{1}, \quad \gamma \rightarrow \gamma^{\prime}=\gamma+\frac{1}{2}\left(b_{1}+(D-2) b_{2}\right),
$$

while there are induced also some total derivative terms of the form (in what follows by " $\delta "$ we denote the corresponding change in the field under a redefinition (8))

$$
\begin{aligned}
& \delta S_{\text {total deriv }}=M_{s}^{D-2} \int d^{D} x \sqrt{-g} \lambda\left(\square g^{\alpha \beta} \delta g_{\alpha \beta}-\nabla^{\nu} \nabla^{\mu} \delta g_{\mu \nu}\right)= \\
& M_{s}^{D-2} \lambda\left(\frac{b_{1}}{2}+(D-1) b_{2}\right) \int d^{D} x \sqrt{-g} \square\left(e^{-\frac{4 \Phi}{\sqrt{3(D-2)}}} R\right), \quad \lambda=\frac{\alpha^{\prime}}{8 g_{s}^{2}} .
\end{aligned}
$$


Such boundary terms are usually dropped, but in our case, where there are domain wall boundaries (branes) in the bulk geometry the terms (10) may play an important rôle in inducing four-dimensional Einstein curvature terms, as well as cosmological constant ("brane tension") contributions on the brane boundaries. This will be the topic of the next section, where such terms will be analysed in some detail.

\section{Warped Geometries, Ambiguities and Induced Cur- vature and Brane Tension on the Boundaries}

In the five-dimensional bulk case, with brane boundaries, it has been shown in [10] that the Randall-Sundrum (RS) warped geometries [1] were solutions of string inspired effective field theories of the type (4)

$$
d s^{2}=e^{-2 \sigma(z)} g_{i j}^{(4)}(x) d x^{i} d x^{j}+d z^{2},
$$

with

$$
\sigma(z)=\sigma_{0}+k|z|
$$

where $\sigma_{0}$ a constant, $x_{i}, i=0, \ldots 3$, are the four-dimensional spacetime coordinates, $g_{i j}^{(4)}(x)$ is the four-dimensional metric, depending only on the four-dimensional coordinates, and $z$ is the extra (bulk) fifth dimension. The presence of the dilaton fields (even if they were constant in the solution) and of the higher-curvature corrections in the action (4) implied the important restriction that the Randall-Sundrum parameter $k$ appearing in the warp factor of the metric, is proportional to the string coupling $g_{s}$, with a proportionality factor of order one. The string coupling was determined by the exponential of the (constant) dilaton field, $g_{s}=e^{\Phi_{0}}$. It was shown in [10] that this was an exact solution of the GaussBonnet corrected gravitational theory (4). Other exact solutions, include dilatonic domain walls, in which there are bulk logarithmic singularities in the metric and the dilaton field, namely the warp factor and the dilaton acquire the form

$$
\sigma(z)=\sigma_{0}+\sigma_{1} \ln \left|1-\frac{z}{z_{s}}\right|, \quad \Phi(z)=\phi_{0}-\frac{3}{2} \ln \left|1-\frac{z}{z_{s}}\right|,
$$

where $z_{s}$ the position of the naked singularities, and $\sigma_{1}$ a numerical constant. The requirement of finiteness of the Planck mass and induced cosmological constant on the brane, required certain restrictions on the domain of $\sigma_{1}$ [10].

The presence of domain walls restricts dynamically the available bulk space. In the simplest scenario, we shall discuss below, the observable world is a brane which is viewed either as a single boundary of a bulk anti-de-Sitter space time, which arises naturally as a solution of the relevant equations of motion, or as one of the two boundaries of the bulk space, the other being the domain wall singularity. 
In either case, the boundary contributions from the bulk field redefinitions (10) result in induced curvature terms and brane tension contributions due to the following observation: Observers on brane worlds will have to integrate over the coordinate $z$ in order to obtain the effective four-dimensional action. The integrated coefficients of the $R^{(4)}(x)$ terms yield contributions to the four-dimensional mass scale squared, $M_{4}^{2}$, whilst the rest of the terms contribute to the effective four-dimensional vacuum energy. Using the warped five-dimensional metric (11) one obtains

$$
\sqrt{-g} R(x)=\sqrt{-g^{(4)}(x)}\left(e^{-2 \sigma(z)} R^{(4)}(x)+e^{-4 \sigma(z)} \mathcal{R}\right)
$$

where

$$
\mathcal{R}=4\left(5 \sigma^{\prime}(z)^{2}-2 \sigma^{\prime \prime}(z)\right)
$$

and the superscript (4) denotes four-dimensional quantities, evaluated on the brane worlds.

On the other hand, the Gauss-Bonnet curvature combinations yield terms of the form [10]

$$
\begin{aligned}
\lambda e^{-\frac{4}{3} \Phi(z)} \sqrt{-g} R_{G B}(x)= & \sqrt{-g^{(4)}(x)} \lambda e^{-\frac{4}{3} \Phi(z)}\left(4 e^{-2 \sigma(z)}\left(3 \sigma^{\prime}(z)^{2}-2 \sigma^{\prime \prime}(z)\right) R^{(4)}(x)\right. \\
& \left.-2\left(R_{\mu \nu \rho \sigma}^{2}-R_{\mu \nu}^{2}\right)+e^{-4 \sigma(z)} \mathcal{R}_{G B}\right),
\end{aligned}
$$

where

$$
\mathcal{R}_{G B}=24\left(5 \sigma^{\prime}(z)^{4}-4 \sigma^{\prime}(z)^{2} \sigma^{\prime \prime}(z)\right)
$$

while dilaton derivative terms in the bulk action contribute

$$
\sqrt{-g}\left(\nabla_{\mu} \Phi\right)^{2}=\sqrt{-g^{(4)}(x)}\left(e^{-4 \sigma(z)} \Phi^{\prime}(z)^{2}+e^{-2 \sigma(z)}\left(\nabla_{i} \Phi^{(4)}(x)\right)^{2}\right) .
$$

In our work here we shall ignore four-dimensional dilaton contributions on the brane world for brevity.

We now observe that, due to (14), the boundary contributions (10), arising from the bulk field redefinitions which cast the bulk action in a Gauss-Bonnet form, yield

$$
\begin{aligned}
\delta S_{\text {boundary }}= & M_{s}^{3} \lambda\left(\frac{b_{1}}{2}+(D-1) b_{2}\right) \int d^{4} x \sqrt{-g^{(4)}(x)} \\
& \left(\left[-2 \sigma^{\prime}(0)-\frac{4}{3} \Phi^{\prime}(0)\right] e^{-2 \sigma(0)-\frac{4}{3} \Phi(0)} R^{(4)}(x)\right. \\
& +8 e^{-4 \sigma(0)-\frac{4}{3} \Phi(0)}\left(-10\left(\sigma^{\prime}(0)\right)^{3}+9 \sigma^{\prime}(0) \sigma^{\prime \prime}(0)-\sigma^{\prime \prime \prime}(0)\right) \\
& \left.-\frac{16 \Phi^{\prime}(0)}{3} e^{-4 \sigma(0)-\frac{4}{3} \Phi(0)}\left(5 \sigma^{\prime}(z)^{2}-2 \sigma^{\prime \prime}(z)\right)\right)
\end{aligned}
$$

from which we see that there are contributions to the induced four-dimensional curvature and the brane tension both proportional to the $b_{1}$ and $b_{2}$ coefficients. 
In our case, by performing the appropriate field redefinitions (8) with $b_{1}, b_{2} \neq 0$ only, we arrive at a Gauss-Bonnet form of the curvature square terms, which themselves yield additional contributions (16) to the induced curvature on the brane $R^{(4)}$, as well as to the brane tension. From the combination of such terms and (14), one obtains the expression for the four-dimensional mass $M_{4}$, as perceived by an observer living on the brane world located at (finite) $z$

$$
\begin{aligned}
& M_{4}^{2}=M_{s}^{3} \int_{-\infty}^{\infty} d z e^{-2 \sigma(z)}\left(1-4 \lambda e^{-\frac{4}{3} \Phi(z)}\left(3\left(\sigma^{\prime}(z)\right)^{2}-2 \sigma^{\prime \prime}(z)\right)\right)+ \\
& M_{s}^{3} \lambda\left(\frac{b_{1}}{2}+(D-1) b_{2}\right)\left[-2 \sigma^{\prime}(0)-\frac{4}{3} \Phi^{\prime}(0)\right] e^{-2 \sigma(0)-\frac{4}{3} \Phi(0)}, \quad \lambda=\frac{1}{8 M_{s}^{2} g_{s}^{2}} .
\end{aligned}
$$

where the last line includes the pertinent field-redefinition-induced boundary contributions (19). It is understood, that the requirement of positivity of the left hand side of the above equation leads to non trivial restriction in the range of the various parameters involved.

In this framework, the four-dimensional effective vacuum energy on the observable brane world $\Lambda_{\text {total }}$ receives two kinds of contributions: (i) from the tension of the brane world we are living on, located, say, at $z=z_{i}=0, V_{\text {brane }}\left(z_{i}\right) \equiv e^{\omega \Phi\left(z_{i}\right)} v\left(z_{i}\right)$, and (ii) from the bulk terms in the action (1), that include the cosmological constant $\xi$, the dilaton derivative terms, as well as the $\mathcal{R}, \mathcal{R}_{G B}$ dependent terms in (14), (16). Therefore, the expression for the total four-dimensional vacuum energy, including the appropriate boundary contribution (19), reads:

$$
\begin{aligned}
& \Lambda_{\text {total }}(0)=\Omega+V_{\text {brane }}(0) \\
& \Omega=\int_{-\infty}^{+\infty} d z e^{-4 \sigma(z)}\left[\xi e^{\frac{4}{3} \Phi(z)}-\frac{4}{3}\left(\Phi^{\prime}(z)\right)^{2}-20\left(\sigma^{\prime}(z)\right)^{2}+8 \sigma^{\prime \prime}(z)+\right. \\
& \left.\lambda e^{-\frac{4}{3} \Phi(z)}\left(24\left(5\left(\sigma^{\prime}(z)\right)^{4}-4\left(\sigma^{\prime}(z)\right)^{2} \sigma^{\prime \prime}(z)\right)+c_{2}\left(\Phi^{\prime}(z)\right)^{4}\right)\right] \\
& V_{\text {brane }}(0)=e^{\omega \Phi(0)} v_{i}+ \\
& 8 M_{s}^{3} \lambda\left(\frac{b_{1}}{2}+(D-1) b_{2}\right) e^{-4 \sigma(0)-\frac{4}{3} \Phi(0)}\left\{\left(-10\left(\sigma^{\prime}(0)\right)^{3}+9 \sigma^{\prime}(0) \sigma^{\prime \prime}(0)-\sigma^{\prime \prime \prime}(0)\right)-\right. \\
& \left.\frac{16 \Phi^{\prime}(0)}{3}\left(5 \sigma^{\prime}(z)^{2}-2 \sigma^{\prime \prime}(z)\right)\right\} .
\end{aligned}
$$

In physically acceptable situations, the quantities $M_{4}^{2}$ and $\Lambda_{\text {total }}$ should be finite, which, in the case in which one encounters bulk singularities, implies certain integrability conditions, as discussed in [10]. This is an important restriction on model building. For the logarithmic solutions (13), for instance, the above requirements, when applied to the $b_{i^{-}}$ independent parts of $\Lambda$ and $M_{4}^{2}$ only, imply

$$
\sigma_{1}<-\frac{1}{4} .
$$

The dependence of physical quantities, such as brane vacuum energies and the fourdimensional mass, on redefinition coefficients gives them a degree of arbitrariness. Indeed, 
the combination of field-redefinition coefficients appearing in (20), would indicate that the relation between the Planck mass and the string mass (scale) is not fixed once an exact solution is given, but depends on the redefinition coefficients $b_{1}$ and $b_{2}$. Moreover, as can be seen from the formulae above, it is not possible to absorb these coefficients in suitable redefinitions of constants in the effective action. One therefore should insist that $b$-dependent terms should define another energy scale when an exact solution of warped geometry is imposed not determined by the parameters of the theory. Such a requirement would in general impose further restrictions on the form of exact solutions of [10], which is a very interesting feature to be discussed next.

\section{Induced Curvature on the Brane and the Need for a New Energy Scale}

A brane world model of high curvature terms in the bulk and induced curvature terms on the brane should be characterized by the following fundamental parameters: three energy scales, i.e. the fundamental Planck mass $M_{5}$ (or bulk scale $M_{s}$ in our five-dimensional toy-string model), the induced-gravity crossover energy scale $r_{c}^{-1}$, and the Gauss-Bonnet coupling energy scale $\lambda^{-1 / 2}=2 \sqrt{2} g_{s} / \sqrt{\alpha^{\prime}}$, and two vacuum energies, i.e. the bulk cosmological constant $\xi$ and the brane tension $V_{\text {brane }}(0)$. These parameters would determine any physical process on the brane, as its cosmological evolution [11].

Had we ignored the surface terms (19) which are induced on the brane because of the freedom we have to do field redefinitions to the graviton and dilaton fields in the bulk, the Gauss-Bonnet term projected on the brane would give a general form for the fourdimensional gravity mass scale on the brane

$$
M_{4}^{2}=M_{s}^{3} \int_{-\infty}^{\infty} d z e^{-2 \sigma(z)}\left(1-4 \lambda e^{-\frac{4}{3} \Phi(z)}\left(3\left(\sigma^{\prime}(z)\right)^{2}-2 \sigma^{\prime \prime}(z)\right)\right) .
$$

If a solution of the background metric (11) is given and the boundary conditions on the brane are satisfied then, the above relation defines the physical four-dimensional Planck mass on the brane in terms of the five-dimensional mass $M_{s}$. If the surface terms (19) are included there is another contribution to the four-dimensional mass

$$
M_{s}^{3} \lambda\left(\frac{b_{1}}{2}+(D-1) b_{2}\right)\left[-2 \sigma^{\prime}(0)-\frac{4}{3} \Phi^{\prime}(0)\right] e^{-2 \sigma(0)-\frac{4}{3} \Phi(0)} .
$$

Even if the background solution is given this relation does not fix the relation between $M_{4}$ and $M_{5}$, introducing another scale in the model. If we define

$$
r_{c}=\frac{M_{4}^{2}}{M_{s}^{3}}=\lambda\left(\frac{b_{1}}{2}+(D-1) b_{2}\right)\left[2 \sigma^{\prime}(0)+\frac{4}{3} \Phi^{\prime}(0)\right] e^{-2 \sigma(0)-\frac{4}{3} \Phi(0)},
$$

we recover the energy scale of induced gravity, known as crossover scale. The contribution of the surface terms to the vacuum energy in relation (21) can also be expressed in terms of the crossover scale. 
Depending on the specific solution one considers, the expressions for the crossover scale and brane tension are different. For instance, in the Randall-Sundrum exact solution [1], the dilaton is a constant, while $\sigma^{\prime}(0)=k=1 / 2 \sqrt{3 \lambda} \propto g_{s} / \sqrt{\alpha^{\prime}}$ as a result of the highercurvature corrections [10]. All the higher $z$-derivatives of $\sigma(z)$ vanish in the RandallSundrum solution. In that case, the crossover scale (and also the brane tension) depend only on the linear combination $\left(\frac{b_{1}}{2}+(D-1) b_{2}\right)$ of the ambiguous coefficients.

On the other hand, in the logarithmic domain-wall solution (13), we have: $\sigma(0)=\sigma_{1} / z_{s}$ (and similarly for its higher $z$-derivatives), and thus one has a dependence on the position of the domain wall brane $z_{s}$ as well as the parameter $\sigma_{1}$ of the solution.

The dependence of physical quantities, such as the crossover scale and the induced brane tension on the field-redefinition ambiguous parameters $b_{i}$ implies of course a "breakdown" of the spirit (but not the letter) of the equivalence theorem in the case of branes in the following sense: although the low-energy effective closed string theory, which lives in the bulk, might enjoy on its own such redefinition ambiguities, in the sense that it is defined perturbatively through the respective scattering amplitudes, which are insensitive to these redefinitions (thus the "letter" of the equivalence theorem holds), nevertheless the presence of brane boundaries, with non trivial string matter on them, leads - through boundary contributions- to a dependence of physical quantities of the brane worlds on these coefficients (thereby leading to an obvious violation of the "spirit" of the theorem).

\section{Conclusions and Discussion}

In this work we have argued that in string theory curvature terms in the field-theoretic effective (low-energy) bulk actions induce scalar curvature and brane tension terms on the boundary.

This happens as a result of the field redefinition ambiguities that characterise the effective actions constructed out of bulk closed string-states scattering amplitudes. The ghost-free Gauss-Bonnet combination, which from a field theoretic point of view is unique, is not special in string theory, precisely as a result of the fact that the underlying string model is always ghost free. In terms of perturbative scattering amplitudes this is achieved by the fact that one can always perform field redefinitions that can cast the target-space higher curvature terms in the effective action in the Gauss-Bonnet combination.

However, in the presence of brane boundaries, this procedure leaves non-trivial curvature and induced brane tension terms, which depend on the ambiguous coefficients. Such ambiguities characterise the crossover scale, as well as the brane tension, and as such cannot be ignored. On the contrary, they are partially fixed by relating the resulting crossover scale with the four dimensional Planck mass, and the induced brane tension with current values of a positive cosmological constant set by astrophysical observations.

An interesting issue concerns the explanation of such ambiguities in the crossover scale from a $\sigma$-model viewpoint. Indeed, it is well known [15] that the local field redefinitions in the bulk string theory correspond to renormalisation-group scheme changes in the corresponding bulk-closed-string $\sigma$-model, amounting to adding local counterterms in the cor- 
responding $\sigma$-model action. Under such scheme changes, the world-sheet renormalisationgroup $\beta$-functions (or rather the so-called Weyl anomaly coefficients [15]) change with a "Lie-derivative" in theory space, the latter being defined by the background $\sigma$-model couplings/fields $g^{i}$

$$
g^{i} \rightarrow g^{i}+\delta g^{i}, \quad \beta^{i} \rightarrow \beta^{i}=\beta^{i}+\delta g^{j} \partial_{j} \beta^{i}-\beta^{j} \partial_{j} \delta g^{i},
$$

where we used infinitesimal field redefinitions for brevity (this situation is representative of our situation, in which the redefinition parameter is provided by the string Regge slope $\left.\alpha^{\prime} \rightarrow 0\right)$. In ordinary string theory, conformal invariance, in the sense of the vanishing of the $\beta^{i}=0$, in a given scheme, is equivalent to string equations of motion $\delta S\left[g^{\prime}\right] / \delta g^{i^{\prime}}=0$ in another scheme (denoted by a prime, $g^{i^{\prime}}$ ), in the sense that there is always a "relative" scheme choice $\delta g^{i}$ which guarantee this.

Let us see how this situation is modified in our case. From the point of view of an open string living on the brane, there is an open $\sigma$-model describing string excitations of the brane world. When a bulk closed string crosses the brane boundary, it may split [20] into two open strings, with their ends attached on the boundary. From a conformal field theory view point such a splitting may be described by the approach of a world-sheet bulk vertex operator $O$ of a closed string, corresponding to, say, a graviton excitation, to a world-sheet boundary $\partial \Sigma$ (parametrised by a real coordinate $s$ ). In such a case one has a novel operator product expansion [20]

$$
O(z, \bar{z} ; s) \sim \sum_{I}(2 s)^{\Delta_{I}-\Delta_{0}} C_{O, \mathcal{E}_{I}}^{A} \mathcal{E}_{I}(s)
$$

provided that the set of boundary conditions $A$ does not break conformal symmetry. Above, $z, \bar{z}$ denote bulk world sheet coordinates, $\Delta$ are the corresponding scaling dimensions of the vertex operators, and $\mathcal{E}_{I}$ denotes a complete set of boundary operators. The splitting amplitudes $C_{O, \mathcal{E}_{I}}^{A}$ can be expressed [20] in terms of bulk operator product expansion (o.p.e.) coefficients $c_{j k}^{i}$, appearing in the bulk $\sigma$-model Weyl anomaly coefficients $\beta^{i}=y^{i} g^{i}+$ $c_{j k}^{i} g^{j} g^{k}+\ldots$ The o.p.e. coefficients appear in the string effective action $[g]$, since the latter is related to the off-shell $\beta$-functions by means of a gradient flow relation

$$
\beta^{i}=\mathcal{G}^{i j} \frac{\delta S[g]}{\delta g^{j}},
$$

with $\mathcal{G}^{i j}$ Zamolodchikov's metric in theory space, related to the two-point function of the corresponding vertex operators.

From this point of view, a bulk redefinition of $g^{i}$ affects the coefficients $c_{j k}^{i}$, according to what was mentioned above, and hence it will affect the splitting amplitudes $C_{O, \mathcal{E}_{I}}^{A}$, thereby inducing local counterterms in the $\sigma$-model describing the world-sheet boundary conformal field theory. In this context, the effective four-dimensional action on the brane world corresponds to the effective target-space action of a $\sigma$-model on the brane propagating on four-dimensional metric backgrounds. In in view of the above-described o.p.e. 
bulk/boundary relations, this effective action depends in general on the splitting amplitudes, since new world-sheet short-distance divergencies arise. This is the root of the violation of the spirit of the equivalence theorem by means of the brane boundary terms.

Although the bare string tension $1 / 4 \pi \alpha^{\prime}$ is the same between the bulk and boundary string theory, nevertheless the effective four-dimensional target space gravitational scale appearing in front of the Einstein term in the four-dimensional target space effective action need not be the same. Indeed, in view of the above-mentioned induction of local counterterms in the boundary $\sigma$-model theory, as a result of renormalisation group scheme changes in the bulk $\sigma$-model, one would obtain a redefinition of the four-dimensional gravitational scale, appearing in front of the Einstein term of the four-dimensional effective action, as well as in a renormalisation of the brane tension itself, as we have seen in previous sections.

The appearance of ambiguities in the brane vacuum energy (induced cosmological constant) brings up another important issue. Namely, an (open) string in a spacetime with a cosmological constant is not in general conformal invariant, and thus an appropriate procedure should be implemented to restore the conformal symmetry. One way to resolve it is to insist on a fine tuned cancellation of the brane cosmological constant by means, e.g. of supersymmetry. The other, and more natural from our point of view, is to dress the boundary $\sigma$-model theory by means of a Liouville field [18]. The spacetime signature of the latter depends on the signature of the induced vacuum energy.

In some of the examples we discussed, it is possible to chose families of solutions of the higher curvature gravity such that the induced vacuum energy on the brane is free from the field redefinition ambiguities. For instance, for the domain wall solutions, this can be achieved by requiring the coefficient of the ambiguous term in the vacuum energy to vanish. This leads to a cubic algebraic equation appearing in (21), which has always a real solution. In fact in this case it can be checked that this solution also satisfies the physical constrain (22). This would ensure a definite sign for the induced vacuum energy of the brane, fixed by the Gauss Bonnet combination [10], which can then be dealt with either via Liouville dressing, or through the traditional Fischler-Susskind mechanism [21], according to which higher-order topologies on the world sheet (string loops) of the open strings on the brane can be held responsible for the appearance of a cosmological constant. Notice that for the Randall-Sundrum solution, it is not possible to choose the coefficient of the ambiguous term in the vacuum energy to vanish.

These considerations cast some doubt on the predictability of brane effective field theory models, embedded in a string theory framework. In string theory, the string bulk scale $M_{s}$ is an arbitrary parameter, and according to our considerations above, this seems to be also the case of the four-dimensional induced brane scale as well as the vacuum energy on the brane world. It may well be that such fundamental issues are not resolved until a fully non-perturbative string/brane theory is available. 


\section{Acknowledgments}

We acknowledge informative discussions with Naresh Dadhich and Alex Kehagias. This work is partially supported by funds made available by the Empeirikeion Foundation (Greece) and by the Greek Ministry of Education (Research program "Pythagoras"). N.M. wishes to thank the National Technical University of Athens for hospitality, during which this work was started and E.P. wishes to thank the Physics Department of King's College London, for hospitality during the last stages of this work.

\section{References}

[1] L. Randall and R. Sundrum, Phys. Rev. Lett. 83, 3370 (1999) [arXiv:hep-ph/9905221], L. Randall and R. Sundrum, Phys. Rev. Lett. 83, 4690 (1999) [arXiv:hep-th/9906064].

[2] P. Binetruy, C. Deffayet and D. Langlois, Nucl. Phys. B 565 (2000) 269 [arXiv:hepth/9905012], N. Kaloper, Phys. Rev. D 60 (1999) 123506 [arXiv:hep-th/9905210], C. Csaki, M. Graesser, C. F. Kolda and J. Terning, Phys. Lett. B 462 (1999) 34 [arXiv:hep-ph/9906513], J. M. Cline, C. Grojean and G. Servant, Phys. Rev. Lett. 83 (1999) 4245 [arXiv:hep-ph/9906523], P. Kanti, I. I. Kogan, K. A. Olive and M. Pospelov, Phys. Lett. B 468, 31 (1999) [arXiv:hep-ph/9909481], P. Kraus, JHEP 9912 (1999) 011 [arXiv:hep-th/9910149], A. Kehagias and E. Kiritsis, JHEP 9911 (1999) 022 [arXiv:hep-th/9910174], P. Binetruy, C. Deffayet, U. Ellwanger and D. Langlois, Phys. Lett. B 477 (2000) 285 [arXiv:hep-th/9910219], D. Ida, JHEP 0009 (2000) 014 [arXiv:gr-qc/9912002], R. Maartens, Phys. Rev. D 62 (2000) 084023 [arXiv:hepth/0004166], P. Bowcock, C. Charmousis and R. Gregory, Class. Quant. Grav. 17, 4745 (2000) [arXiv:hep-th/0007177].

[3] J. Garriga and T. Tanaka, Phys. Rev. Lett. 84, 2778 (2000) [arXiv:hep-th/9911055].

[4] N. Arkani-Hamed, S. Dimopoulos and G. R. Dvali, Phys. Lett. B 429 (1998) 263 [arXiv:hep-ph/9803315], I. Antoniadis, N. Arkani-Hamed, S. Dimopoulos and G. R. Dvali, Phys. Lett. B 436 (1998) 257 [arXiv:hep-ph/9804398], N. ArkaniHamed, S. Dimopoulos and G. R. Dvali, Phys. Rev. D 59 (1999) 086004 [arXiv:hep$\mathrm{ph} / 9807344]$.

[5] P. Binetruy, C. Deffayet and D. Langlois, Nucl. Phys. B 565, 269 (2000) [arXiv:hepth/9905012]; for reviews see: D. Langlois, Prog. Theor. Phys. Suppl. 148, 181 (2003) [arXiv:hep-th/0209261]; R. Maartens, Living Rev. Rel. 7, 1 (2004) [arXiv:grqc/0312059]. E. Papantonopoulos, [arXiv:gr-qc/0410032], U. Gunther and A. Zhuk, [arXiv:gr-qc/0410130].

[6] J. M. Maldacena, Adv. Theor. Math. Phys. 2, 231 (1998) [Int. J. Theor. Phys. 38, 1113 (1999)] [arXiv:hep-th/9711200]. 
[7] G. R. Dvali, G. Gabadadze, M. Kolanovic and F. Nitti, Phys. Rev. D 64, 084004 (2001) [arXiv:hep-ph/0102216]; G. R. Dvali and G. Gabadadze, Phys. Rev. D 63, 065007 (2001) [arXiv:hep-th/0008054].

[8] G. R. Dvali, G. Gabadadze and M. Porrati, Phys. Lett. B 485, 208 (2000) [arXiv:hepth/0005016].

[9] E. Papantonopoulos and V. Zamarias, JHEP 0410, 051 (2004) [arXiv:hepth/0408227].

[10] N. E. Mavromatos and J. Rizos, Phys. Rev. D 62, 124004 (2000) [arXiv:hepth/0008074]; Int. J. Mod. Phys. A 18, 57 (2003) [arXiv:hep-th/0205299].

[11] G. Kofinas, R. Maartens and E. Papantonopoulos, JHEP 0310, 066 (2003) [arXiv:hepth/0307138].

[12] S. C. Davis, Phys. Rev. D 67, 024030 (2003) [arXiv:hep-th/0208205]; E. Gravanis and S. Willison, Phys. Lett. B 562, 118 (2003) [arXiv:hep-th/0209076].

[13] C. Charmousis and J. F. Dufaux, Class. Quant. Grav. 19, 4671 (2002) [arXiv:hepth/0202107].

[14] S. Nojiri and S. D. Odintsov, [arXiv:hep-th/0409244].

[15] E.S. Fradkin and A.A. Tseytlin, Nucl. Phys. B262(1985)1; D.J. Gross and J.H. Sloan, Nucl. Phys. B291 (1987) 41; R. R. Metsaev and A. A. Tseytlin, Nucl. Phys. B 293, 385 (1987).

[16] M. C. Bento and N. E. Mavromatos, Phys. Lett. B 190, 105 (1987); N. E. Mavromatos and J. L. Miramontes, Phys. Lett. B 201, 473 (1988).

[17] S. S. Gubser, I. R. Klebanov and A. M. Polyakov, Phys. Lett. B428 (1998)105; E. Alvarez and C. Gomez, hep-th/9810102; J. de Boer, E. Verlinde and H. Verlinde, hepth/9912012; E. Verlinde and H. Verlinde, JHEP 0005 (2000) 034, and references therein [hep-th/9912018]; A. Dhar and S. R. Wadia, Nucl. Phys. B 590, 261 (2000) [arXiv:hep-th/0006043]; John Ellis, N.E. Mavromatos, and D.V. Nanopoulos, Erice SubNuclear Series, Vol. 31 (1993), p.1 [hep-th/9403133]; J. Chaos, Solitons and Fractals, 10 (1999), 345 (eds. C. Castro and M.S. El Naschie, Elsevier Science, Pergamon 1999), [hep-th/9805120].

[18] F. David, Mod. Phys. Lett. A3 (1988), 1651; J. Distler and H. Kawai, Nucl.Phys. B321 (1989) 509.

[19] B. Zwiebach, Phys. Lett. B156, (1985) 315.

[20] J. L. Cardy and D. C. Lewellen, Phys. Lett. B 259, 274 (1991). 
[21] W. Fischler and L. Susskind, Phys. Lett. B 171, 383 (1986); Phys. Lett. B 173, 262 (1986). 